\title{
Development of Relative Disparity Sensitivity in Human Visual Cortex
}

\author{
DAnthony M. Norcia, Holly E. Gerhard, and Wesley J. Meredith \\ Department of Psychology, Stanford University, Stanford, California 94305
}

Stereopsis is the primary cue underlying our ability to make fine depth judgments. In adults, depth discriminations are supported largely by relative rather than absolute binocular disparity, and depth is perceived primarily for horizontal rather than vertical disparities. Although human infants begin to exhibit disparity-specific responses between 3 and 5 months of age, it is not known how relative disparity mechanisms develop. Here we show that the specialization for relative disparity is highly immature in 4- to 6-month-old infants but is adult-like in 4- to 7-year-old children. Disparity-tuning functions for horizontal and vertical disparities were measured using the visual evoked potential. Infant relative disparity thresholds, unlike those of adults, were equal for vertical and horizontal disparities. Their horizontal disparity thresholds were a factor of $\sim 10$ higher than adults, but their vertical disparity thresholds differed by a factor of only $\sim 4$. Horizontal relative disparity thresholds for 4 - to 7 -year-old children were comparable with those of adults at $\sim 0.5 \mathrm{arcmin}$. To test whether infant immaturity was due to spatial limitations or insensitivity to interocular correlation, highly suprathreshold horizontal and vertical disparities were presented in alternate regions of the display, and the interocular correlation of the interdigitated regions was varied from $0 \%$ to $100 \%$. This manipulation regulated the availability of coarse-scale relative disparity cues. Adult and infant responses both increased with increasing interocular correlation by similar magnitudes, but adult responses increased much more for horizontal disparities, further evidence for qualitatively immature stereopsis based on relative disparity at 4-6 months of age.

Key words: binocular vision; maturation; stereopsis; visual evoked potentials

\section{Significance Statement}

Stereopsis, our ability to sense depth from horizontal image disparity, is among the finest spatial discriminations made by the primate visual system. Fine stereoscopic depth discriminations depend critically on comparisons of disparity relationships in the image that are supported by relative disparity cues rather than the estimation of single, absolute disparities. Very young human and macaque infants are sensitive to absolute disparity, but no previous study has specifically studied the development of relative disparity sensitivity, a hallmark feature of adult stereopsis. Here, using high-density EEG recordings, we show that 4- to 6-monthold infants display both quantitative and qualitative response immaturities for relative disparity information. Relative disparity responses are adult-like no later than $4-7$ years of age.

\section{Introduction}

Retinal disparity created by the lateral separation of the eyes is first encoded in primary visual cortex. Two properties of the disparity tuning of these cells, however, make them unlikely substrates for perceptual depth. First, disparity tuning is present for

Received Nov. 19, 2016; revised April 21, 2017; accepted April 25, 2017.

Author contributions: A.M.N. and H.E.G. designed research; A.M.N., H.E.G., and W.J.M. performed research; A.M.N., H.E.G., and W.J.M. analyzed data; A.M.N. and H.E.G. wrote the paper.

This work was supported by National Institutes of Health, National Eye Institute Grant EY018875. We thank Chuan Hou for an earlier version of this study (Norcia and Hou, 2005), which was presented at the Vision Sciences Society Meeting in 2005.

The authors declare no competing financial interests.

Correspondence should be addressed to Dr. Anthony M. Norcia, Department of Psychology, Stanford University, 450 Serra Mall, Jordan Hall, Building 420, Stanford, CA 94305. E-mail: amnorcia@stanford.edu.

DOI:10.1523/JNEUROSCI.3570-16.2017

Copyright $\odot 2017$ the authors $\quad 0270-6474 / 17 / 375608-12 \$ 15.00 / 0$ dynamic random dot stereograms (DRDSs) whose features are of opposite contrast in the two eyes (Cumming and Parker, 1997). These patterns do not give rise to perceptual depth (Cogan et al., 1993, 1995). Second, cells in V1 are primarily sensitive to absolute disparity: for example, the image disparity defined relative to the fixation plane, but not relative disparity, the difference in disparity between two or more features (Cumming and Parker, 1999; Thomas et al., 2002). The perceptual system, by contrast, is quite insensitive to absolute disparity (Westheimer, 1979; Erkelens and Collewijn, 1985; Cottereau et al., 2012a), and fine depth discrimination relies on a computation of relative disparity (Westheimer, 1979; McKee et al., 1990; Andrews et al., 2001).

The likely substrate for perceptual depth from disparity lies in extrastriate cortex. Cells tuned for relative disparity are first found in V2 of the macaque (Thomas et al., 2002) and have also 
A

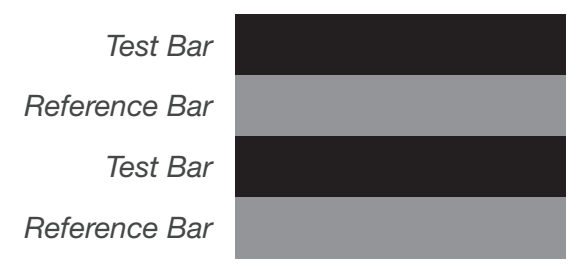

Disparity Swept Correlation $=100 \%$

Disparity $=0$ Correlation $=100 \%$

Disparity Swept Correlation $=100 \%$

Disparity $=0$ Correlation $=100 \%$
B

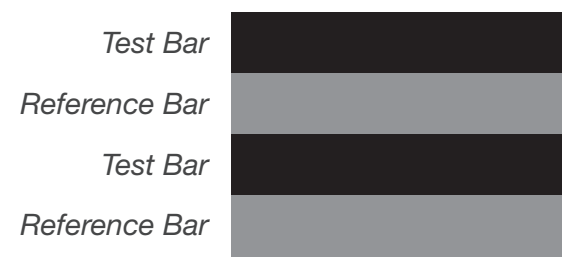

Disparity Fixed Correlation $=100 \%$

Disparity $=0$ Correlation Swept 0-100\%

Disparity Fixed Correlation $=100 \%$

Disparity $=0$ Correlation Swept 0-100\%

Figure 1. Schematic illustration of the stimulus conditions. The display consisted of test and reference bands forming a horizontally oriented cyclopean grating with a square-wave profile in the disparity domain $(0.55 \mathrm{c} / \mathrm{deg})$. $\boldsymbol{A}$, Disparity sweep condition. The disparity was alternated at $2 \mathrm{~Hz}$ between 0 disparity and an age-dependent range of suprathreshold crossed disparities. Reference bars presented a constant 0 disparity, $100 \%$ correlated stimulus. $\boldsymbol{B}$, Interocular correlation sweeps. The disparity of the test bars was alternated at $2 \mathrm{~Hz}$ at an age-dependent value that was well above disparity threshold. This value was constant throughout the trial. The interocular coherence of the reference bars was swept from $0 \%$ to $100 \%$ interocular correlation. The interocularly correlated dots in the reference bars were presented at a fixed and unmodulated disparity of 0 .

been found in V3 (Anzai et al., 2011), V4 (Umeda et al., 2007; Shiozaki et al., 2012), IT (Janssen et al., 2001), and, depending on the precise definition of relative disparity, MT (Krug and Parker, 2011). In parallel with the relative disparity processing hierarchy, disparity tuning that is specific for the matched interocular contrasts that support perceptual depth is increasingly apparent as one goes from V1 to V4 (Tanabe et al., 2004) to IT (Janssen et al., 2003).

Developmentally, cells tuned for absolute disparity have been found as early as $14 \mathrm{~d}$ of age in infant macaque V1 (Chino et al., 1997; Maruko et al., 2008) and V2 (Maruko et al., 2008). In humans, visual evoked potentials (VEPs) can be measured to changes in interocular correlation as early as 2-3 months of age (Braddick et al., 1980; Petrig et al., 1981; Braddick and Atkinson, 1983; Birch and Petrig, 1996; Jandó et al., 2012; Mikó-Baráth et al., 2014). By contrast, the development of fine stereo-acuity, a function that is dependent on the extraction of relative disparity, has a protracted developmental sequence, reaching near adult levels only in school age children (for review, see Norcia and Gerhard, 2015).

Using DRDS to isolate disparity-selective processes and highdensity EEG recordings, we determined the smallest relative disparity that could evoke a synchronized response in 4- to 6-month-old infants, 4- to 7-year-old children, and adults. As a control for the relevance of the measured evoked response for perceptual depth, measurements were also made for vertical disparities that do not support a percept of depth. Adults were a factor of $\sim 4$ more sensitive to horizontal than vertical disparities, but infant sensitivity was approximately equal and was reduced by a factor of $\sim 10$ relative to the adult for horizontal disparities. Relative disparity responses in 4- to 7-year-olds were qualitatively and quantitatively adult-like. The lack of specialization for horizontal relative disparity in infants was confirmed in a second experiment in which we varied the strength of the interocular correlation in a zero-disparity reference region.

\section{Materials and Methods}

Participants. A total of 23 adults between the ages of 19 and 43 years (mean age $=25.5$ years, 12 female) recruited from the Stanford community participated. Their visual acuity was better than 0.1 LogMar in each eye without spectacle correction and they had $<0.1$ LogMar acuity difference between the two eyes. The adult participants also had stereo-acuity of 40 arcsec or better on the Randot test. A total of 25 infants, age range 3.8-6.4 months (mean age $=6$ months, 11 female) and 17 children, age range $4.4-7$ years (mean age $=5.5$ years, 9 female) were recruited via letters mailed to parents on the basis of information provided by the California Department of Vital Statistics. The infants and children had no history of major medical problems, were $>2500$ g at birth, and were presumed to be otherwise typically developing.

Stimulus generation. The experimental stimuli consisted of dynamic random-dot stereograms. Dichoptic viewing was achieved via red/ blue glasses, as described previously (Norcia et al., 2005). The dot luminance was equated for red and blue images viewed through the optics of the glasses, and cross talk was minimal as judged psychophysically. The DRDSs were displayed on a 65 inch Sony Bravia XBR$65 \mathrm{HX} 929$ LCD monitor. The field of view was $40^{\circ} \times 40^{\circ}$ at a viewing distance of $100 \mathrm{~cm}$. The dots were 4.6 arcmin in diameter and were presented at a density of 5 dots per square degree. The dots were drawn with OpenGL using antialiasing at a screen resolution of $1920 \times 1080$. This function allowed us to present disparities via dithering that were smaller than the nominal resolution set by the $1920 \times 1080$ display matrix. This was verified by examining the contents of video memory and through examination of the antialiased pixels under magnification. The dots were updated at $20 \mathrm{~Hz}$.

Experimental procedure. The stimuli are illustrated schematically in Figure 1. In one set of conditions, designed to measure disparity thresholds and suprathreshold voltage versus disparity functions, the disparity presented in alternate bands of a square-wave disparity grating was modulated at $2 \mathrm{~Hz}$ from 0 disparity to one of 10 equal logarithmically spaced crossed disparities (0.5-16 arcmin for adults and children, 2-32 arcmin for infants). The other bands of the disparity grating were presented at 0 disparity throughout. The disparity values were changed once every second as a swept parameter. The trials began with a $1 \mathrm{~s}$ "prelude" in which the display presented the first stimulus value in the disparity sweep. In the correlation sweep conditions, the interocular correlation of the alternating bands was swept between $0 \%$ and $100 \%$ in 10 equal, linearly spaced steps. The disparity of these bands was 0 . The other bands contained a fixed disparity modulation that was between 0 and 16 arcmin for the adults and between 0 and $24 \operatorname{arcmin}(n=9)$ or 0 and $47 \operatorname{arcmin}(n=9)$ for the infants. Not all the infants provided data in both the disparity and correlations sweep conditions.

The adults participated in one recording session lasting $\sim 60 \mathrm{~min}$. The disparity and correlation sweeps were presented in random order with 10 repetitions of each of the four experimental conditions. The infant data were recorded in two sessions, spaced by $\sim 1$ week. Within an infant session, the horizontal and vertical versions of either the disparity or correlation sweep conditions were presented, with the day-order of presentation of the disparity or correlation sweep versions of the experiment being randomized over infants. The children participated in a single session during which 20 trials each of the horizontal and vertical disparity sweep conditions were presented.

EEG acquisition and preprocessing. High-density, 128-channel EEGs were recorded using HydroCell electrode arrays and an Electrical Geodesics NetAmp400. The EEG was sampled natively at $500 \mathrm{~Hz}$ and then 
resampled to $420 \mathrm{~Hz}$, giving exactly 7 datum samples per video frame. The display software provided a digital trigger indicating the start of the trial with millisecond accuracy. The data were filtered to a $0.3-50 \mathrm{~Hz}$ passband upon export of the data to custom signal processing software. Artifact rejection was performed in two steps. First, the continuous filtered data were evaluated according to a sample-by-sample thresholding procedure to locate consistently noisy sensors. These channels were replaced by the average of their six nearest spatial neighbors. Once noisy electrode voltages were interpolated in this fashion, the EEG was rereferenced from the $\mathrm{Cz}$ reference used during the recording to the common average of all the sensors. Finally, EEG epochs that contained a large percentage of data samples exceeding threshold $(30-80 \mu \mathrm{V}$ for adults, $200 \mu \mathrm{V}$ for infants) were excluded on a sensor-by-sensor basis.

EEG data and statistical analysis. The steady-state VEP amplitude and phase at the first four harmonics of the disparity update frequency of $2 \mathrm{~Hz}$ were calculated via a Recursive Least Squares (RLS) adaptive filter (Tang and Norcia, 1995). The RLS filter comprised two weights: one for the imaginary and the other for the real coefficient of each frequency of interest. The RLS filter was updated at $420 \mathrm{~Hz}$, and the learned coefficients were averaged over an exponential forgetting function with a decay parameter of $1 \mathrm{~s}$. Estimates of background EEG noise level during the trials were derived from the same analysis performed at frequencies straddling the harmonic frequency of interest $( \pm 1 \mathrm{~Hz})$. A variant of Hotelling's $T^{2}$ statistic (Pei et al., 2017) was used to test whether the VEP response differed reliably from 0 amplitude.

Dimension reduction via reliable components analysis. To reduce the dimensionality of the high-density steady-state VEP data to a small number of physiologically interpretable components, we used a component decomposition technique, reliable components analysis (RCA), which is based on trial-to-trial phase consistency (Dmochowski et al., 2015). Briefly, the method exploits the fundamental underlying assumption of steady-state VEPs that response phase is constant over repeated presentations of the same stimulus. This assumption is instantiated by the crosstrial covariance matrix, which is decomposed by solving a generalized eigenvalue problem into a small number of maximally reliable components consisting of a scalp topography and response spectrum.

\section{Results}

\section{Disparity response functions}

As we have shown in previous work, periodic modulation of disparity at $2 \mathrm{~Hz}$ evokes scalp responses at several harmonics of the disparity-update frequency (e.g., at 2, 4, 6, and $8 \mathrm{~Hz}$ ), with the first harmonic being the largest in amplitude (Cottereau et al., 2011, 2012a, b). The scalp distribution for the most reliable component ( $\mathrm{RC} 1)$ is shown in Figure 2 for adults and infants. RCA was performed to reduce the dimensionality of the data to perform a principled analysis of response components, especially in terms of the selection of electrodes for analysis. The RC1 scalp topography learned over the first four harmonics of the disparityupdate frequency was maximal over midline occipital electrodes in both adults and infants. The topography appears to be shifted somewhat dorsally on the recording array for adults relative to that for infants; but given the uncertainty in how the scalp array overlays underlying cortex, we do not interpret this further.

As a first basis of comparison of infant and adult disparity response functions, we begin with the first harmonic component (1F1), which our previous work has shown to be a sensitive indicator of relative disparity processing in a range of retinotopic visual areas (Cottereau et al., 2011, 2012a, b). In adults, the disparity response function for horizontal disparity, shown as Figure 2 (top right, blue trace), rises monotonically from the noise level (dashed line), with the first run of statistically significant responses being recorded at $1.17 \mathrm{arcmin}$. In the following, isolated bins with $p<0.05$ are not considered as indicating the appearance of the response above the noise level, but runs of two or more consecutively significant bins are. This noise-level-based
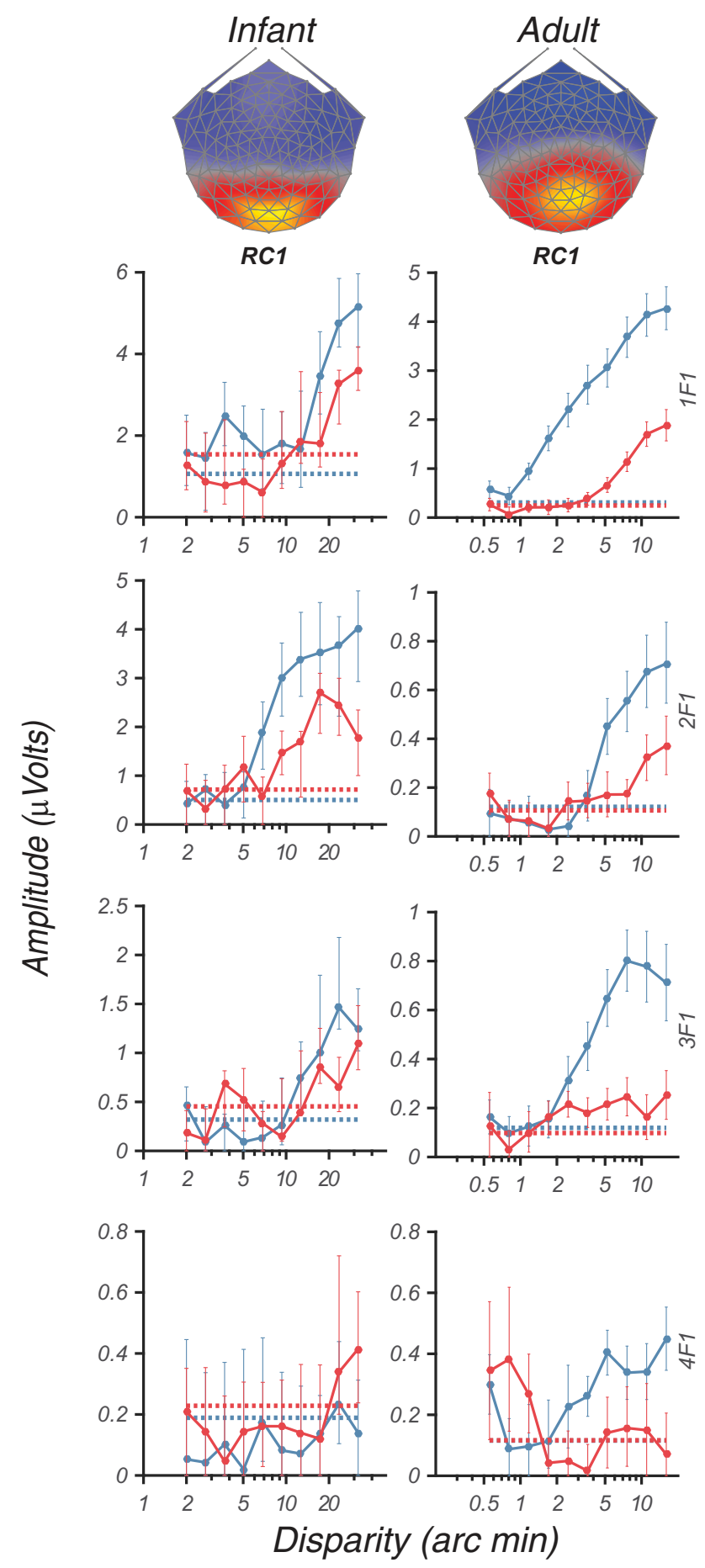

Figure 2. RC1 disparity sweep responses. Top, Response topographies for the RC1 components are maximal over occipital mid-line electrodes in both infants (left) and adults (right). Bottom, Evoked response amplitude as a function of disparity (semilog axes) for infants (left) and adults (right). Blue curves plot data recorded with horizontal disparity. Red curves plot data for vertical disparity. Infant responses are of comparable magnitude at $1 F 1$ and $2 F 1$, but adult responses are larger at $1 F 1$. Differences between horizontal and vertical disparity responses are large in adults but not infants, and adults are more responsive to the smallest disparities.

criterion for defining the disparity threshold is useful but underestimates visual sensitivity (e.g., the inverse of the disparity threshold) because the experimental noise level, which is a combination of background EEG, myogenic, and instrumental noise, limits the detectability of small visually driven responses. An al- 
ternative method for threshold/sensitivity estimation involves extrapolation of the response function to zero amplitude (Campbell and Maffei, 1970; Norcia et al., 1989). Threshold measured by extrapolation to zero amplitude was $0.46 \mathrm{arcmin}$.

The adult response function for vertical disparities (red trace) does not begin to rise above the noise level until 4-5 arcmin versus $\sim 1$ arcmin for horizontal disparity and the corresponding zero-amplitude threshold was 1.74 arcmin versus 0.46 arcmin. Above threshold, the peak amplitude of the horizontal disparity response is larger than that for the vertical disparity condition by a factor of 2, and the form of the functions is largely consistent with a pure rightward shift along the disparity axis. Adults thus show larger evoked responses that have a lower threshold for horizontal disparities than for vertical disparities.

Infants, by contrast, are overall less sensitive to disparity. Their $1 \mathrm{~F} 1$ response functions only rise above the noise level at 17 arcmin for both horizontal and vertical disparities (Fig. 2, left). A total of 18 infants contributed data, and the mean age at the time of recording was 4.7 months (range 3.8-5.5 months, SD 0.4 months; 8 female). The extrapolated threshold for horizontal disparity is 4.5 arcmin, a factor of $\sim 10$ higher than in adults. The vertical disparity response threshold ( $17 \mathrm{arcmin})$ is a factor of $\sim 4$ higher than for adults. In terms of the relative thresholds for horizontal versus vertical disparity, infants are 1.6 times more sensitive to horizontal disparity, but adults are a factor of 3 times more sensitive. At suprathreshold disparities, the relative peak amplitude for horizontal versus vertical disparity was 1.4 for infants versus a factor of 2 for adults. The dominant component of the adult relative disparity response is thus immature quantitatively as indicated by the higher disparity values that are needed to drive measurable responses and qualitatively because the difference in sensitivity between horizontal and vertical disparities is less pronounced.

\section{Higher harmonic responses for horizontal and vertical disparity}

In adults, the most reliable component ( $\mathrm{RC} 1)$ contains measurable responses up to at least the fourth harmonic $(4 \mathrm{~F} 1,8 \mathrm{~Hz})$ for horizontal disparities and 3F1 for vertical disparities (Fig. 2, right). For horizontal disparities, the $2 \mathrm{~F} 1 / 4 \mathrm{~Hz}$ component rises above the noise at 5.2 arcmin, a value that is a factor of 3 higher than needed to drive a measurable response at $1 \mathrm{~F} 1 / 2 \mathrm{~Hz}$. The corresponding values are also 5.2 arcmin for $3 \mathrm{~F} 1$ and $4 \mathrm{~F} 1$. The peak response amplitude occurs at the largest disparity for each harmonic, and the 1F1 component has the largest peak amplitude, with $1 \mathrm{~F} 1$ being 5.2 times larger than 2F1, 4.1 times larger than $3 \mathrm{~F} 1$, and 9 times larger than $4 \mathrm{~F} 1$.

In infants, $2 \mathrm{~F} 1 / 4 \mathrm{~Hz}$ component of $\mathrm{RC} 1$ is relatively more prominent than their $1 \mathrm{~F} 1 / 2 \mathrm{~Hz}$ component: $1 \mathrm{~F} 1$ in infants is only a factor of 1.3 larger than $2 \mathrm{~F} 1$, instead of being a factor of 5.2 larger, as in adults. The infants' $2 \mathrm{~F} 1$ response for horizontal disparities also becomes measurable at a smaller disparity value than for 1F1 ( 9 arcmin vs 17 arcmin), which is opposite to what is observed in adults where $1 \mathrm{~F} 1$ rises out of the noise at a lower value than does $2 \mathrm{~F} 1$. The difference in the magnitude of horizontal disparity needed to evoke a measurable response is smaller in the infants for $2 \mathrm{~F} 1$ (9 vs $5.2 \mathrm{arcmin}$ ) than it is for $1 \mathrm{~F} 1$ (17 vs 1.7 arcmin), but it is still elevated relative to the adult value. The dominance of the infant response by even harmonics suggests that their responses may be more driven by disparity change than by the appearance/disappearance of the disparity defined bars (Cottereau et al., 2012b).

Harmonic components above $2 \mathrm{~F} 1$ are not reliable in infants for either RC1 or RC2 components. This result, combined with the different relative amplitudes of $1 \mathrm{~F} 1$ and $2 \mathrm{~F} 1$, indicates that the infants' response dynamics differ from those of the adults, with adults having relatively more sustained activity as captured by the larger relative contribution of $1 \mathrm{~F} 1$ to their response. Together, the infant response to disparity displays both spatial sensitivity and dynamical immaturities.

\section{Disparity tuning of the RC2 component}

The component decomposition indicated that a second component (RC2) was present in both infants and adults. The basis of the component extraction is trial-by-trial covariance of the response, and components are thus expected to index distinct underlying cortical sources. That this is the case is borne out by the different scalp topography of RC1 and RC2 and their different sensitivity to horizontal and vertical disparity. The scalp topography of RC2 differs between adults and infants being approximately symmetric, bilaterally in adults, but skewed to the right hemisphere in infants (compare Fig. 3, left vs right columns; the component polarity is arbitrary). In both adults and infants, RC1 is focally distributed on the occipital mid-line, whereas RC2 is more broadly and posteriorly distributed.

Like RC1, RC2 vertical disparity responses were much smaller than horizontal disparity responses in adults and were, indeed, unmeasurable (compare Fig. 3, red traces with those of Fig. 2). Infants, on the other hand, had small but measurable 1F1 vertical disparity responses as part of their RC2 component, but their horizontal disparity response were unmeasurable, a pattern opposite that of the adults. At 2F1, horizontal disparities produced measurable responses in both adults and infants. The sensitivity of the infant 2F1 horizontal and vertical disparity response components was similar to each other, unlike the case for adults where no measurable response was recorded for vertical disparities. Here again, as was the case for the RC1 component, the RC2 component shows a preferential response to horizontal disparity in adults but not in infants.

\section{Interocular correlation response functions}

The first recordings showed that 4- to 6-month-old infants are less sensitive to disparity than are adults and that their disparity system does not display the characteristic superiority of horizontal over vertical disparities near threshold. In the second set of recordings, we asked whether this insensitivity to disparity comes about from a more general lack of sensitivity to interocular correlation rather than limitations that are purely spatial, such as those that would come about from a relative lack of high-spatial frequency contrast sensitivity or the availability of small receptive fields (Legge and Gu, 1989; Harris et al., 1997; Filippini and Banks, 2009). That is, in situations where the fineness of the disparity cue is not limiting performance, is disparity processing adult-like? To examine this question, we presented a changing disparity stimulus that was well above the infant and adult disparity threshold in one set of bars and then varied the interocular correlation in the neighboring bars. By doing this, we could measure the extent to which the evoked response depends on the strength of the interocular correlations in the reference bands (e.g., the quality of the relative disparity cue) (Cottereau et al., $2011,2012 b)$. We can also ask whether this dependency differs for horizontal disparities that are expected to strongly drive the relative disparity system compared with vertical disparities that are not. 

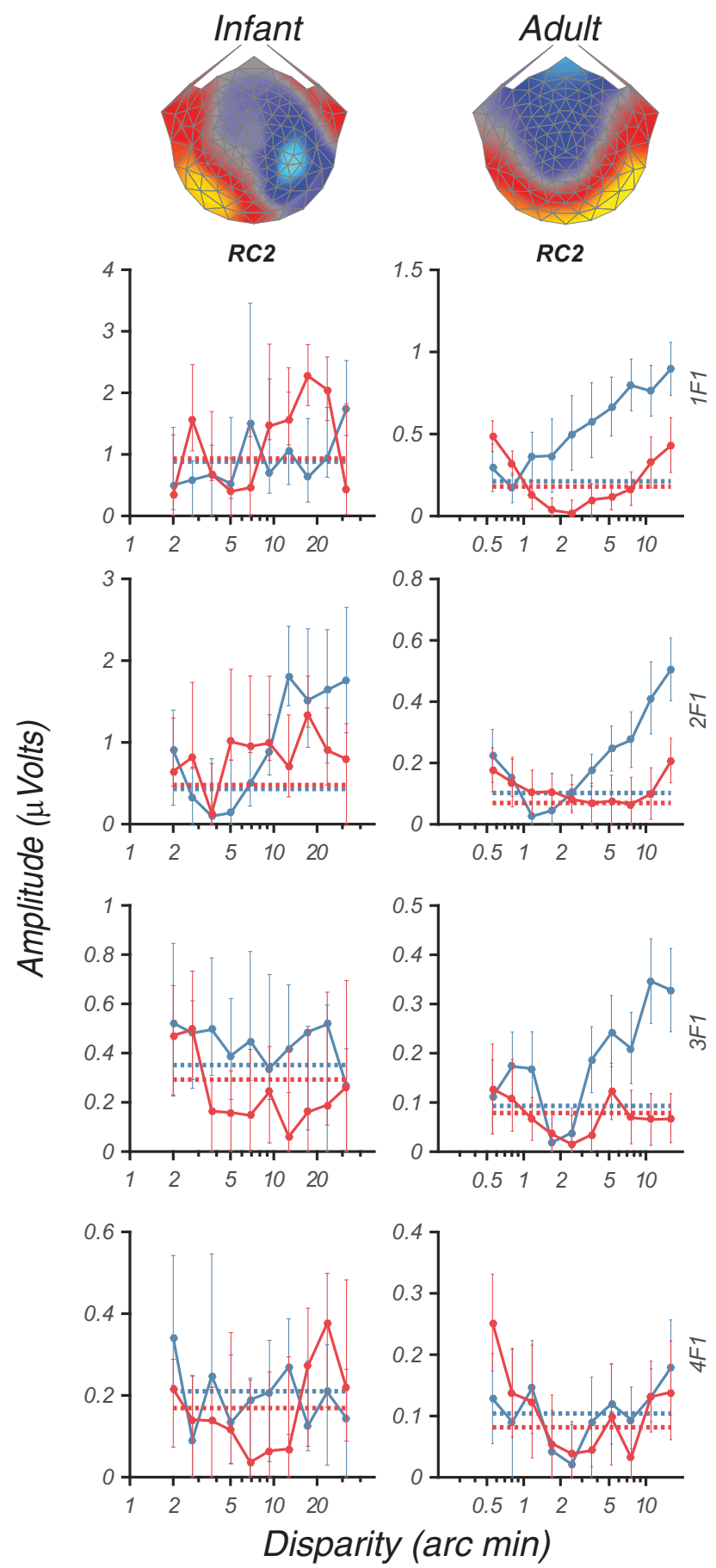

Figure 3. RC2 disparity sweep responses. Top, Infant response topography for the RC2 components has a configuration consistent with a tangential source in dorsal occipital cortex (top left). The adult topography is strongest for electrodes at the edge of the array consistent with ventral surface sources. Bottom, Evoked response amplitude as a function of disparity (semilog axes) for infants (left) and adults (right). Blue curves plot data recorded with horizontal disparity. Red curves plot data for vertical disparity. Infant responses are most reliable at 2F1 but do not clearly differ for horizontal and vertical disparities. Adult responses, when above the noise level (dotted lines), are larger for horizontal disparities (e.g., at 1F1, 2F1, and 3F1).

\section{First harmonic horizontal and vertical correlation response functions}

We describe the data from this experiment in the same order as we described the data from the disparity response function experiment, beginning with RC1 and the dominant first harmonic component of the adults and infants. The $\mathrm{RC} 1$ higher harmonic responses are then described followed by the results from RC2. Components were derived from the independent data collected in this experiment.

The evoked responses for the RC1 component are shown as a function of interocular correlation in Figure 4. In adults (Fig. 4, right), the stimulus presented in the first bin of the interocular correlation trials consisted of a 16 arcmin changing disparity with zero interocular correlation in the reference bars. This bin thus serves as a baseline against which to measure the effects of increasing the quality of the relative disparity information in the display. Responses locked to the disparity alternation rate in the first bin should not be the result of relative disparity processing with respect to the reference bars because the dots comprising these bars are uncorrelated between the two eyes. There is nonetheless a measurable response in the first bin that could arise from a number of sources. First and most simply, this response could be driven by changes in absolute disparity (Cottereau et al., 2011, $2012 \mathrm{~b}$ ). It is also possible that the frame of the monitor may serve as a reference, as we have shown referencing effects can extend over considerable distances (Cottereau et al., 2012a).

In adults, the responses in the horizontal disparity condition increase systematically as the interocular correlation in the reference bar increases, being $\sim 4$ times larger when the reference bars are $100 \%$ correlated versus when they are $0 \%$ correlated. For vertical disparities, measurable responses are present at all values of interocular correlation, but the response increases only modestly as a function of interocular correlation (a factor of $1.5 \mathrm{vs}$ a factor of 4; Fig. 4, right, compare red vs blue curves).

In addition to differences in response amplitude as a function of the reference quality, the response dynamics as measured by the phase of the evoked response also differ for horizontal and vertical disparities as shown in Figure 5. Response phase is a composite measure of conduction delays and cortical integration time. The response phase for the vertical disparity condition in adults changes systematically as a function of interocular correlation in the reference bars (Fig. 5, open symbols), but the phase for horizontal disparity does not (Fig. 5, filled symbols). For vertical disparity, the response is at -178 degrees at zero correlation, shifting to -41 degrees at $100 \%$ correlation, a total change in phase of 137 degrees. The phase shift is in the direction of an increasing response delay going from $0 \%$ to $100 \%$ interocular correlation. By contrast, the response phase for horizontal disparities begins at -29 degrees at 0 correlation and shifts by only 17 degrees across all levels of interocular correlation, ending at -46 degrees, a value not significantly different from the asymptotic value for vertical disparity. The horizontal and vertical disparity response phase and amplitude functions are strongly dissociated. Response phase is different when interocular correlation is zero, but it is the same when it is $100 \%$, Response magnitudes are similar at zero interocular correlation, but very different at $100 \%$. There is thus no value of correlation at which the two responses are the same.

Quantitative modeling of the sensitivity of horizontal disparity detectors suggests that they can have residual sensitivity to vertical disparities (Read, 2010), and this may account for the phase shift we observe for vertical disparities under the following assumption. As interocular correlation increases, the responsiveness of horizontal relative disparity mechanisms increases and their residual sensitivity to vertical disparity would increase as a secondary result. This signal may mix with absolute disparity responses to vertical disparity. That horizontal disparity responses are slower than vertical disparity responses in the absence of a reference has not been previously reported. V1 cells do, how- 


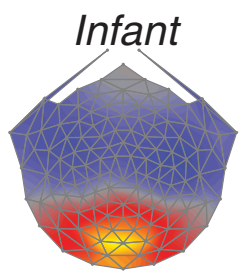

RC1
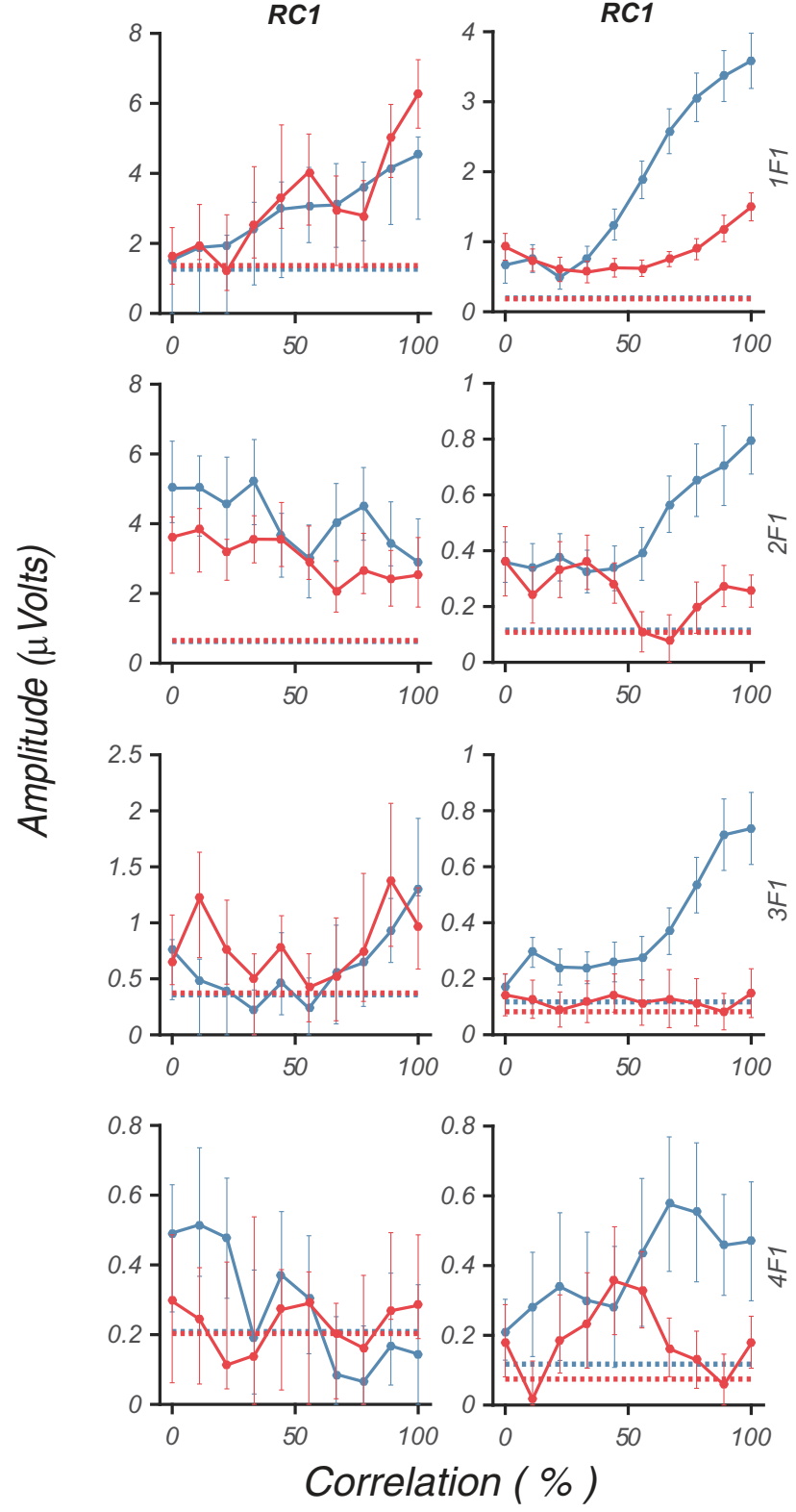

Figure 4. RC1 interocular correlation sweep responses. Top, Response topography for the RC1 component is maximal over occipital mid-line electrodes in both infants (left) and adults (right). Bottom, Evoked response amplitude as a function of interocular correlation (linear axis) for infants (left) and adults (right). Blue curves plot data recorded with horizontal disparity. Red curves plot data for vertical disparity. Infant responses have approximately equal response amplitudes for 1F1 and 1F2 components, neither of which are strongly tuned for disparity orientation. By contrast, the adult response is dominated by $1 \mathrm{~F} 1$ and is strongly tuned for disparity orientation. Dotted lines indicate the noise level measured at neighboring frequencies.

ever, show a specialization for horizontal disparity that is not related to relative disparity processing (Cumming, 2002).

The pattern of response at each harmonic in the infants differs from that of the adults, further indicating functional immatu-

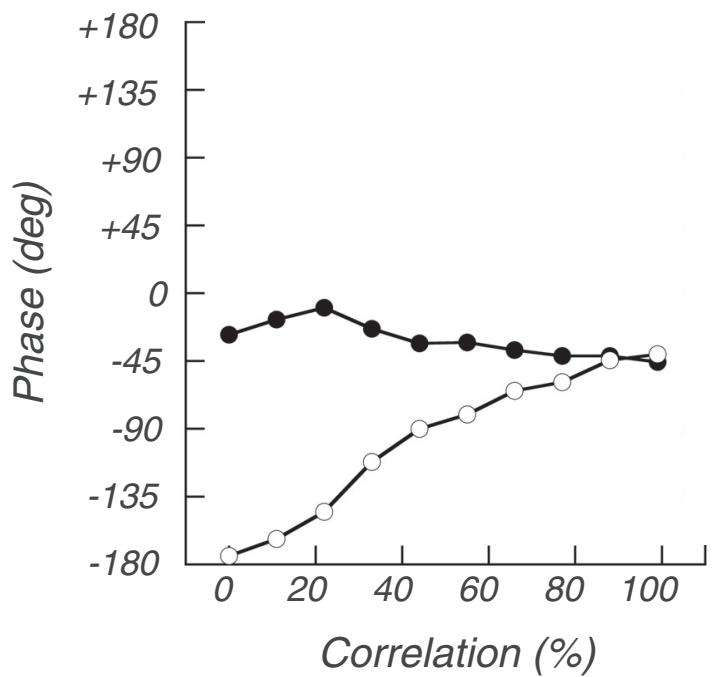

Figure 5. Temporal response phase for horizontal disparities in adults, measured at 1 F1 for RC1. Response phase shifts in the direction of decreasing phase lag between $0 \%$ and $100 \%$ interocular correlation for vertical disparity (open symbols), but not for horizontal disparity (filled symbols).

rities in their disparity processing system (Fig. 4, left panels). Eighteen infants contributed data, including 11 participants from the disparity sweep condition. Their mean age was 5.0 months (range 4.3-6.4 months; SD 0.5 months). Unlike in the adult, the infant $1 \mathrm{~F} 1$ component increases by approximately the same amount for both horizontal and vertical disparities compared with the unreferenced value measured from the first bin (factors of 3 and 3.9, respectively, for infants vs 4 and 1.5 for adults). The results at $2 \mathrm{~F} 1$ also indicate a relative lack of specialization for horizontal relative disparities. Both groups have a measurable response in the first bin of the sweep where the interocular correlation in the reference bars is zero. These responses likely reflect responses to cells tuned for absolute disparity. In adults, the correlation-related response increases systematically for horizontal, but not for vertical, disparities. For infants, the $2 \mathrm{~F} 1$ response decreases, rather than increases, as interocular correlation increases for both horizontal and vertical disparities. Thus, the infant response pattern at $2 \mathrm{~F} 1$ bears little resemblance to that of the adult either in the form of the tuning function or in the relationship between horizontal and vertical disparity tuning. As noted for the disparity response functions, the adult response contains activity at 3F1 and 4F1, but only for horizontal disparities. There is a small but measurable $3 \mathrm{~F} 1$ response in the infants that is of comparable magnitude for horizontal and vertical disparities, but no measurable response at $4 \mathrm{~F} 1$.

\section{RC2 component of the interocular correlation response function}

The topography of the RC2 component in adults measured during the correlation sweeps was similar to that recorded for disparity sweeps (compare Fig. 6, right, with Fig. 3, right), indicating that a similar set of underlying generators is being tapped in the two stimulus regimens. The infant topography differs somewhat from that measured from the disparity sweep condition but still retains the right hemisphere asymmetry seen in those measurements.

Overall, disparity responses are less robust for RC2 than for $\mathrm{RC} 1$. This is manifested in terms of sensitivity to interocular correlation and the relative absence of responses at higher harmonics 


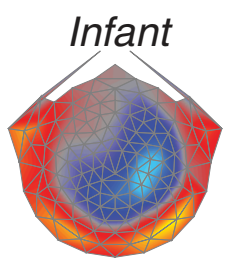

RC2
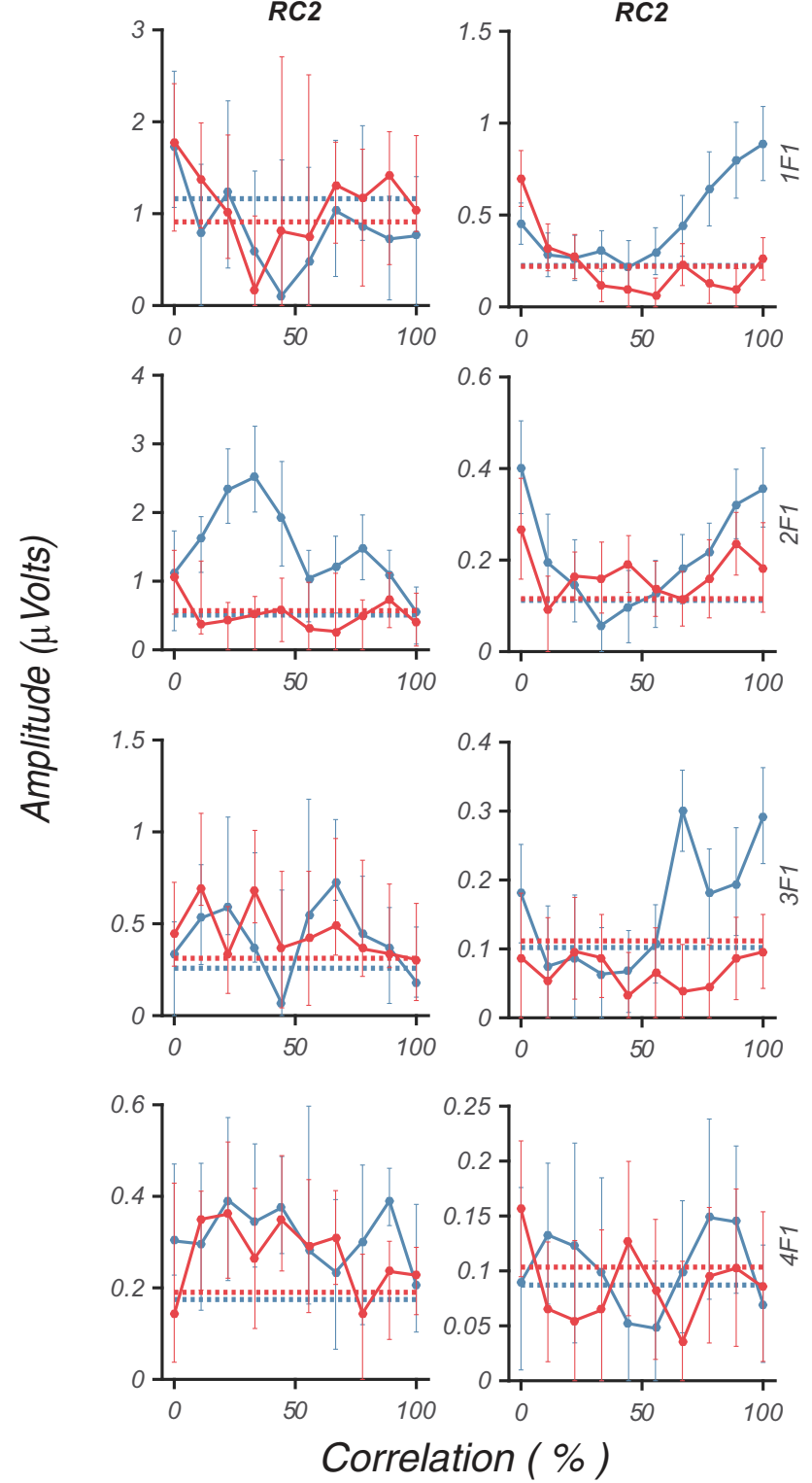

Figure 6. RC2 interocular correlation sweep responses. Top, Response topography for the RC2 components is maximal over occipital mid-line electrodes in both infants (left) and adults (right). The map polarity is arbitrary. Bottom, Evoked response amplitude as a function of interocular correlation (linear axis) for infants (left) and adults (right). Blue curves plot data recorded with horizontal disparity. Red curves plot data for vertical disparity. Infant responses are dominated by the 2F1 component, whereas the adult response is dominated by the 1F1 component. Responses, when above the noise level (dotted lines), are larger for horizontal disparities.

and for vertical disparities. First, the 1F1 component of RC2 in adults is less sensitive to interocular correlation than is the same component of RC1 (zero amplitude thresholds of $8.8 \pm 3.7 \%$ vs $30 \pm 9 \%)$. Second, there are no reliable responses to vertical disparity for RC2, unlike the case for RC1 where there was a clear response to vertical disparities at $1 \mathrm{~F} 1$. RC2 thus indexes a process in the adults that responds preferentially to horizontal disparities. Finally, there is no response at $4 \mathrm{~F} 1$ for RC2, but there is for RC1.

In the infants, there is no measurable $1 \mathrm{~F} 1$ component for $\mathrm{RC} 2$, unlike the case for RC1 where both horizontal and vertical disparities evoked responses with comparable amplitudes and sensitivities (compare Fig. 6, left with Fig. 4, left). The infant 2F1 response function, by contrast, peaks over this portion of the correlation response function, but only for horizontal disparities. The relative specificity of RC2 for horizontal disparity is thus reinforced by the infant data. The $2 \mathrm{~F} 1$ component of RC2 is an isolated example of a specialization for horizontal disparity in infants.

\section{Disparity tuning in 5- to 7-year-olds}

Prior behavioral studies of stereo-acuity development have found that disparity thresholds for a range of targets approach adult levels in 5- to 7-year-olds (Norcia and Gerhard, 2015). We therefore decided to record from children of this age to not only compare their disparity thresholds to those of adults with the same apparatus but also to determine whether the dynamic aspects of the response and its topographic distribution were also mature at this age. Because the clearest immaturities in terms of disparity sensitivity were found with the disparity tuning functions, rather than the correlation function, we focused our measurements on the former.

The response topography for RC1 in children is very similar to that of the adults, peaking at occipital midline electrodes (compare Fig. 7, top left with Fig. 2, top right). The horizontal disparity tuning function for RC1, 1F1 (Fig. 7, left, blue curve) rises above the noise level at $1.17 \mathrm{arcmin}$, as it did for the adults. The disparity threshold was 0.55 arcmin, which is comparable with that of the adults and consistent with the children's performance on the Frisby stereo-acuity test. However, the disparity response function for both $1 \mathrm{~F} 1$ and $2 \mathrm{~F} 1$ components of $\mathrm{RC} 1$ is not a simple nearly linear function of log disparity, as in adults. The child response functions have saturating portions, but the adult response functions are each monotonically increasing.

Because the children and adults viewed exactly the same stimulus, it is also possible to directly compare response phase in addition to response amplitude. The phase versus disparity behavior of the 1F1 component differs substantially between the children and adults. In both children and adults, the response becomes reliable at 1.17 arcmin and thus phase can be estimated. These data are shown in Figure 8. In adults, response phase is -5 degrees at 1.17 arcmin and progresses in the phase lead (decreasing delay) direction, asymptoting at -44 degrees. The total phase shift of 39 degrees is equivalent to a speeding of the response by 54 ms between threshold and 16 arcmin. In the children, the phase of the response near threshold ( $1.17 \mathrm{arcmin})$ is 54 degrees. This phase value is consistent with the response to small disparities being $81 \mathrm{~ms}$ slower compared with the adults. The child function asymptotes at -37 degrees versus -40 degrees, as it does in the adults, a relative latency difference of only $4 \mathrm{~ms}$ for highly suprathreshold disparities. Thus, although the children are equally sensitive to fine horizontal disparities, their responses over the finest disparity range are delayed.

As in adults, the children's vertical disparity response at $1 \mathrm{~F} 1$ is smaller than that for horizontal disparity (Fig. 7, left, blue traces). There are indeed no consistent runs of significant response for their vertical disparity response functions for any harmonic component of $\mathrm{RC} 1$. The children have a robust $2 \mathrm{~F} 1$ response for horizontal disparity and a much weaker response to vertical dis- 


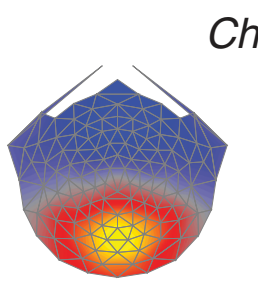

Children
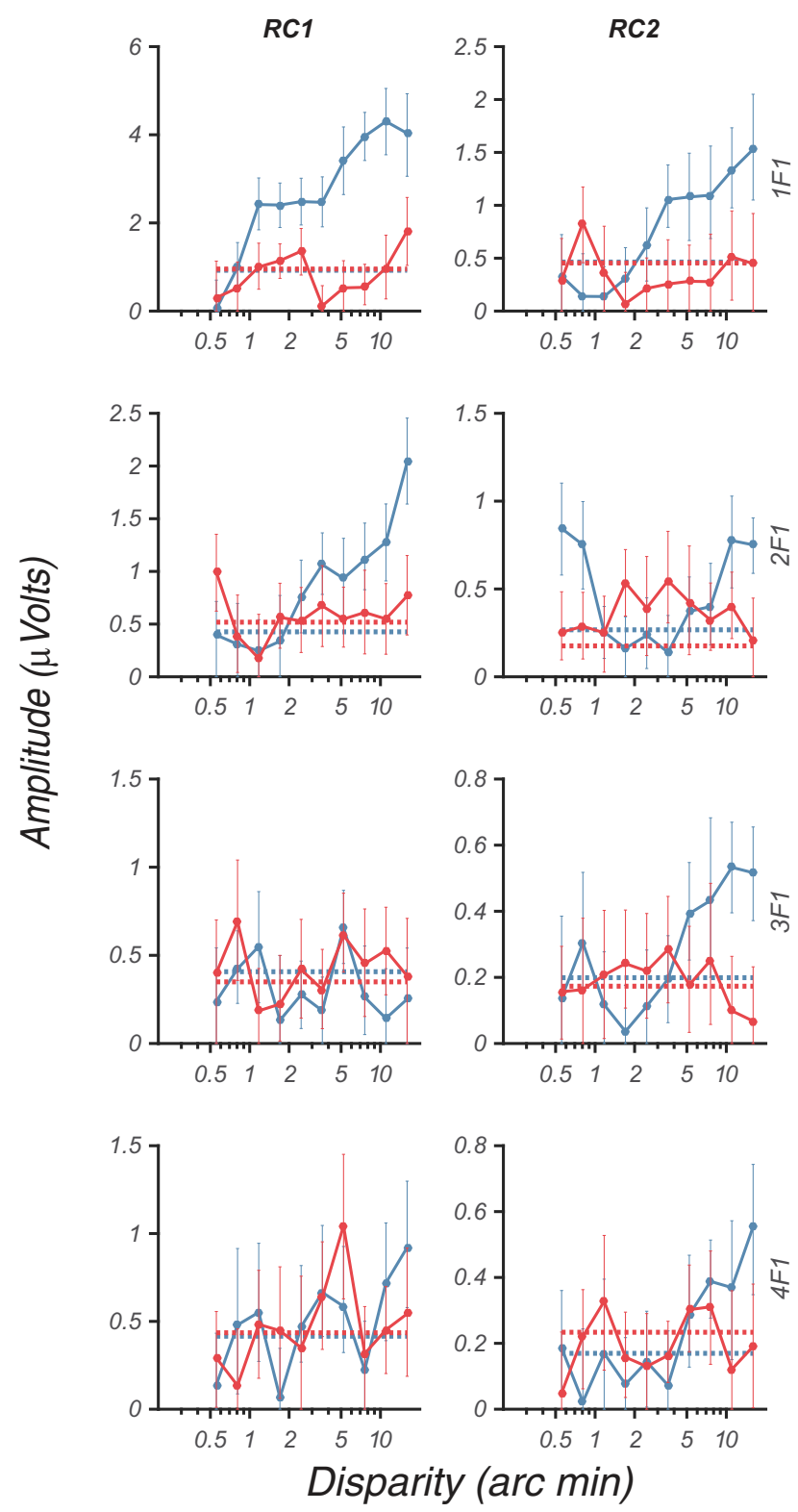

Figure 7. Disparity response functions for 4- to 7-year-old children. Disparity sensitivity is approximately the same as that of adults, and the children show a clear distinction between horizontal and vertical disparities. There is some suggestion of a double-limbed response function, rather than a simple monotonically increasing function, as in the adults. The noise level during the trials (horizontal dotted line) was measured as the mean of the response at frequencies $1 \mathrm{~Hz}$ below and $1 \mathrm{~Hz}$ above the response frequency.

parity, as was the case for adults and for infants. Like the infants, the third and fourth harmonic responses are very weak but are clearly measurable in the adults (compare Fig. 7, left with Fig. 3, right). This result, combined with the relatively delayed responses near threshold, suggests that the dynamics of the evoked response to disparity must continue to develop after age 7 .

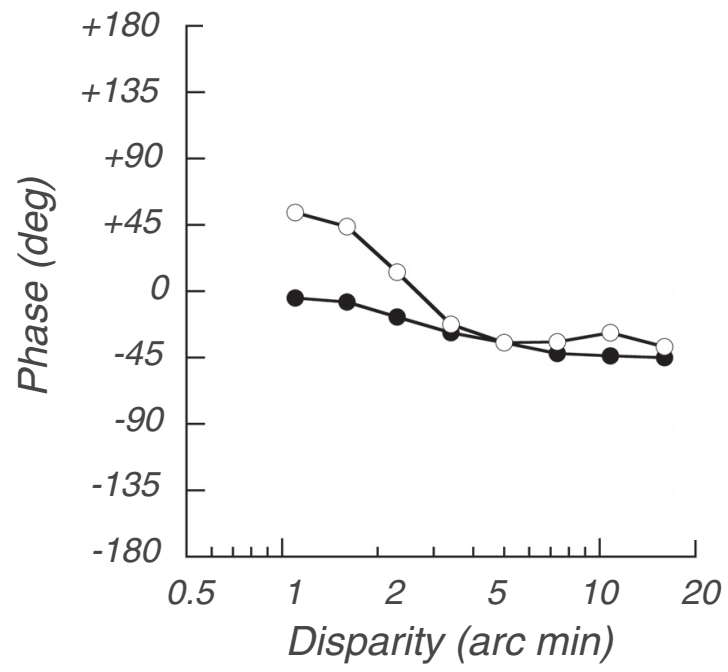

Figure 8. Horizontal disparity response temporal phase measured at $1 F 1$ for children (open symbols) and adults (filled symbols) for the RC1 component. Child response phase lags that of the adults for small disparities.

Turning to RC2, the children's topography (Fig. 7, right), like the adults, has bilateral maxima (the color/polarity of the component maps is arbitrary up to an inversion), again suggesting that the RC1 and RC2 reflect the activity of different underlying sources. The child RC2, 1F1 component for horizontal disparity (blue traces) becomes measurable starting at $1.17 \operatorname{arcmin}(p=$ 0.003 as was the case for their $1 \mathrm{~F} 1$ component of RC1). There is no measurable $1 \mathrm{~F} 1$ response for vertical disparity (red traces) for the RC2 component. This is a general trend across all of our analyses: the RC2 response to vertical disparity is much weaker than it is for horizontal disparity. RC2 may reflect a higher stage of processing where perceptually relevant disparities have been selected for processing. This is consistent with the topographic distribution of this component, which is bilateral, rather than being centered on the occipital pole.

\section{Responses at the dot-update frequency}

Because of the high temporal resolution of the EEG, it is possible to record time/phase-locked responses elicited by the updating of the random dot fields at $20 \mathrm{~Hz}$. For notational purposes, we refer to these responses as the $1 \mathrm{~F} 2$ component, indicating that they are first harmonic responses to the second temporal frequency in the display, F2. These responses could be related to luminance change or to contrast change, as both these properties update at this rate. Consistent with this, responses at the dot-update rate can be recorded with dynamic random dot patterns that are viewed monocularly (Eizenman et al., 1999). Alternatively, F2-related responses could also be due to binocular processing as the match between corresponding points in the display must be reestablished on every update as all of the dots have changed.

The scalp topography of the most reliable component of the dot-update response measured at 1F2 was maximal over midline occipital electrodes and resembled that for RC1 of the disparity response function data in the infants and adults (compare Fig. $9 A$, top, $C$, top with Fig. 2, top left and Fig. 2, right, respectively). The 1F2 topography is similar for the children (Fig. 9B). The topographies for the 1F2 component measured during the interocular correlation sweeps are similar to those measured during the disparity sweeps, especially within an age group (compare Fig. 

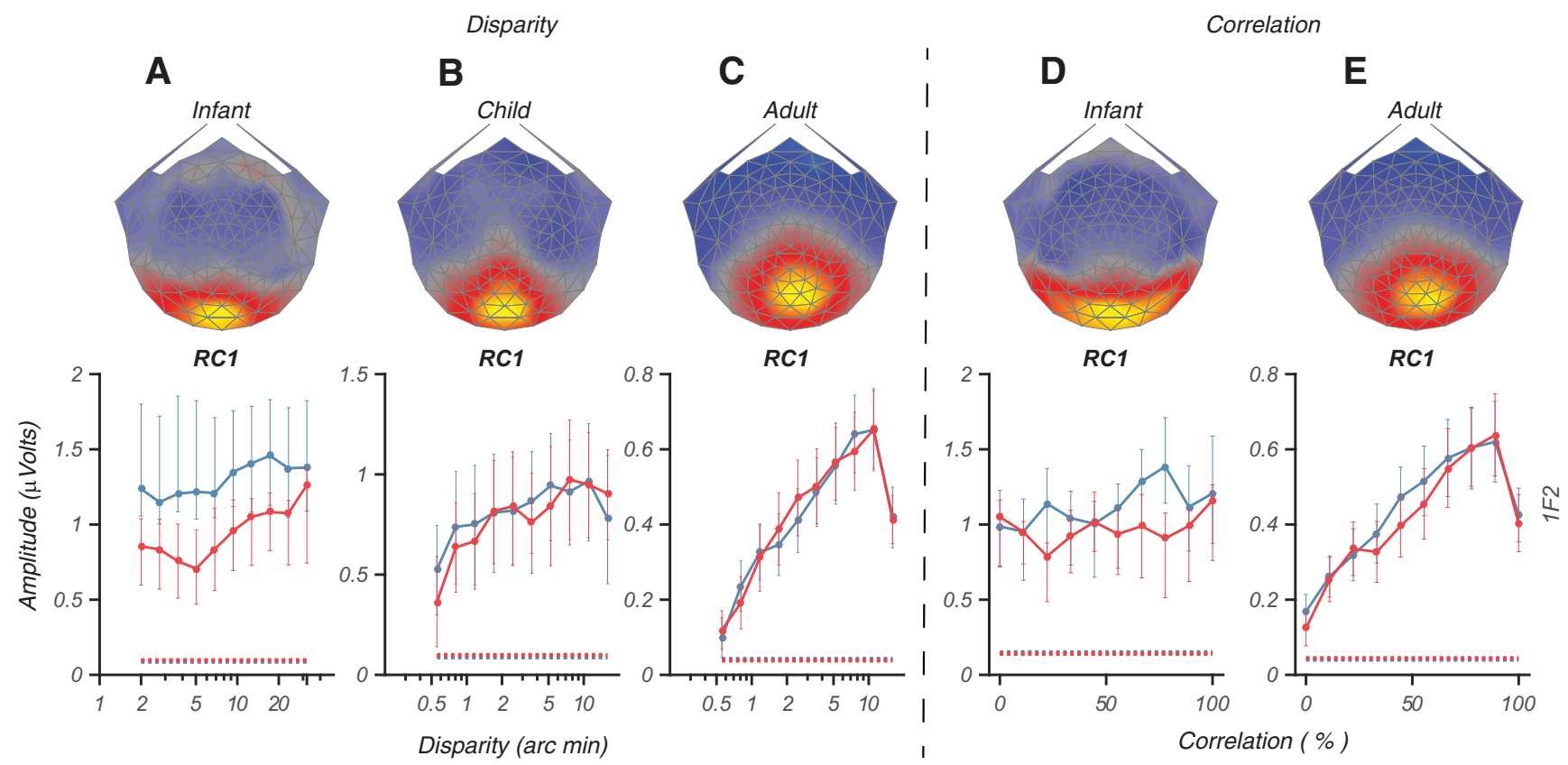

Figure 9. Responses at 1F2, the dot-update rate (20 Hz). $\boldsymbol{A}, \boldsymbol{B}$, and $\boldsymbol{C}, 1 \mathrm{~F} 2$ amplitude as a function of disparity for infants, children, and adults. Blue curves plot data for horizontal disparity conditions. Red curves plot data for vertical disparity conditions. The adult response function is strongly tuned compared with that of the infants, and both functions are independent of disparity orientation. The adult function increases in amplitude up to 11 arcmin and then decreases at 16 arcmin. The child response function has an intermediate slope and is also independent of disparity orientation. $\boldsymbol{D}$ and $\boldsymbol{E}, 1 \mathrm{~F} 2$ amplitude as a function of interocular correlation. The adult response function is strongly tuned with amplitude increasing as a function of interocular correlation up to $90 \%$, with a decrease at $100 \%$ interocular correlation. Infant response amplitudes are largely independent of interocular correlation. Red and blue horizontal lines indicate the corresponding background EEG noise levels during the trials, measured as the mean of the response at 19 and $21 \mathrm{~Hz}$, respectively, averaged over the $10 \mathrm{~s}$ of the sweep trial.

$9 A$, top with Fig. $9 D$, top, and Fig. $9 B$ with Fig. $9 E$ ). The topographies are consistent with sources in early visual cortex.

The infant response functions at $1 \mathrm{~F} 2$ show little dependence on either disparity (Fig. 9A) or interocular correlation (Fig. 9D). Within a sweep type, there is no difference between horizontal and vertical disparities. By contrast, response functions measured during the disparity sweep condition in adults are strongly dependent on the magnitude of the disparity and somewhat less so in the children (Fig. 9B, C). Interestingly, unlike the case for 1F1 and its harmonics, there is no difference in response profile for horizontal and vertical disparities at any age.

The magnitude of the 1F2 component depends strongly on interocular coherence in the adults (Fig. 9E), but not in the infants (Fig. 9D). Here again, the response does not depend on disparity orientation. In adults, the $1 \mathrm{~F} 2$ response component increases as a function of both disparity and interocular correlation, dropping in amplitude in the last bin of the sweep, which in each case corresponded to a 16 arcmin $100 \%$ correlation stimulus.

The fact that the 1F2 response depends on disparity, at least in children and adults, is consistent with a binocular contribution to this response. Such a binocular contribution could come from cells that make the binocular match between the dots. Because the dots are temporally uncorrelated, the binocular match must be reestablished at $20 \mathrm{~Hz}$. The $1 \mathrm{~F} 2$ component is immature in the infants because it does not show a dependence on either disparity or interocular correlation, as it does in the adults. The fact that the 1F2 response depends on both disparity and interocular correlation, but not disparity orientation in the adults, indicates that it receives a contribution from a different, possibly lower-level binocular mechanism than the one(s) generating responses at the harmonics of the disparity change frequency $(\mathrm{F} 1)$.

\section{Discussion}

High-grade stereopsis in the adult visual system is based on the extraction of horizontal relative disparity information. Here we show that the relative disparity system is both quantitatively and qualitatively immature in 4- to 6-month-old infants whose absolute disparity responses measured in the absence of a disparity reference are robust. The quantitative immaturity manifested as elevations in disparity thresholds and the qualitative immaturity manifested as a lack of specialization for horizontal disparities in a fine stereo-acuity task and in a coarse interocular correlation task, as well as in a lack of disparity tuning of the dot-update response. We find that the relative disparity system is adult-like in its disparity threshold and disparity orientation preference no later than 4-7 years of age, although minor differences in the shape of the disparity tuning functions and response dynamics are still present.

We measured responses to vertical disparity because vertical disparity stereograms, like anticorrelated stereograms, do not give rise to a percept of depth, but they do elicit disparity-tuned responses from single neurons in V1 (Cumming and Parker, 1997; Cumming, 2002; Durand et al., 2007). Differential responsiveness to horizontal versus vertical disparities is a signature of the perceptually relevant disparity system in the same way that differential responsiveness to correlated and anticorrelated stereograms has been used as a probe of the substrate of stereopsis, defined as a percept of depth from disparity. We found that infant responses to vertical disparities were similar to their responses to horizontal disparities for both fine disparities that define the disparity threshold as well as for highly suprathreshold disparities. The generality of these results over a wide range of disparities and values of interocular correlation suggests that the immaturity 
seen in the infants is not simply a quantitative question of sensitivity to fine position information or interocular correlation. Rather, functional specializations specific to horizontal relative disparities are selectively immature.

\section{Relationship to previous studies of the development of stereopsis}

This is the first study in any species to specifically examine the development of relative disparity processing using a neural measure. The most relevant prior data for comparison to the present results is the substantial number of studies of stereo-acuity development in infants and children (for review, see Norcia and Gerhard, 2015). Stereo-acuity development is relevant here because it is controlled by mechanisms that rely on the presence of relative disparity in the display (Westheimer, 1979; McKee et al., 1990; Andrews et al., 2001). The most comparable previous study in human (Birch and Petrig, 1996) measured infant sensitivity to horizontal disparities using DRDS portraying stripes of alternating crossed and uncrossed disparity. Thresholds for individual infants determined by extrapolation to zero amplitude varied widely. At 5 and 6 months, thresholds ranged from $\sim 2$ arcmin to $\sim 0.15$ arcmin, as did adult thresholds. Extrapolation to zero amplitude is subject to bias toward lower thresholds when the signalto-noise ratio is low and the slope of the regression function is shallow (Norcia et al., 1989). This may have led to the wide range of individual thresholds, including some very low threshold values. Our approach to disparity threshold measurement was to use group-mean response functions based on coherent averages across participants. Using this approach, thresholds were 4.5 arcmin in infants and $\sim 0.5$ arcmin in children and adults; and from this, we conclude that infant sensitivity to horizontal disparity is not at adult levels between 4 and 6 months. The Birch and Petrig (1996) study did not specifically manipulate the availability of relative disparity information as we have done in the second experiment. VEP responses in their study could have arisen from cells that are only sensitive to absolute disparity.

Behavioral stereo-acuity measurements differ widely on the apparent maturity of infant stereopsis. Two studies using static random dot stereograms (Birch and Salomão, 1998; Brown et al., 2007) each found substantial differences between infant and adult stereo-acuity, but two early studies using line stereograms (Held et al., 1980; Birch et al., 1982) reported that stereo-acuity develops from being unmeasurable at 3 months to being fully adult by 6 months ( 1 arcmin). It is not clear to what this strikingly precocious behavior should be attributed.

Behavioral disparity thresholds have been measured in 5- to 7-year-old children on a variety of stereo-acuity tasks (for review, see Simons, 1981; Norcia and Gerhard, 2015) and with a range of criteria for successful discrimination. The general consensus from this work is that stereo-acuity is quite good at 5-7 years of age, but it is not quite at adult levels. Our results are consistent with this view. Our conclusion on this point is strengthened by the fact that the same display parameters and VEP recording and measurement criteria were applied at all ages. Moreover, the VEP imposes a consistent and minimal set of task demands across age, something that is not possible with tasks that require overt behavioral choices and understanding verbal task instructions.

\section{Immaturity persists far above disparity threshold}

Previous analyses (Brown et al., 2007) have suggested, based on measurements of infant contrast sensitivity and simulations in adults, that much of the quantitative difference in stereo-acuity observed between infants and adults may come from differences in their monocular contrast sensitivity. They reduced adult stereo-acuity to infant levels by reducing stimulus contrast by factors of 50- to 80-fold. Three aspects of our results suggest that poor monocular contrast sensitivity is not the only immaturity limiting the infants' relative disparity system. The first is that the adult thresholds for vertical disparity are much higher than those for horizontal disparity, but infants' thresholds are not. An overall increase in contrast sensitivity is not expected to preferentially favor disparity energy filters of a particular orientation. A second line of evidence for qualitative immaturity comes from the manipulation of the interocular correlation in the reference bars in the second experiment. In adults, the evoked response for horizontal disparity depended strongly on the availability of interocularly correlated dots in the reference bars. The response to horizontal and vertical disparity orientations was similar at zero interocular correlation (e.g., for absolute disparity) but diverged as interocular correlation increased and relative disparity cues became available. The adult response to vertical disparity showed only a small effect of the level of interocular correlation in the reference bars, and this sensitivity could have been driven by off-axis responses from the horizontal disparity system (Read, 2010). Infants, by contrast, showed a similar dependency on reference quality for both horizontal and vertical disparities at all values of interocular correlation. The experiment was explicitly designed to minimize the dependence of the measured responses on small spatial mechanisms that could have been immature because of limited monocular acuity of the infant. The lack of a clear disparity orientation effect in the reference-quality measurements further implicates a qualitative immaturity that can be dissociated from limited stereo-acuity.

Finally, responses at the dot-update rate (1F2) depend on both disparity and interocular correlation in the adults, but not in the infants. This suggests that this response component may be monocularly driven in infants. That responses at the dot-update rate depend on disparity has not been reported before, and it is unclear at present what mechanism underlies these responses. The fact that these disparity-sensitive responses do not depend on disparity orientation and that they occur at the rapid rate at which interocular matching occurs suggests that they may arise in early visual cortex and before the functional specialization that selectively emphasizes horizontal disparity arises both anatomically in extrastriate cortex and developmentally. These responses are large for the smallest disparity in infants but are unmeasurable for small disparities in adults. This suggests that monocular aspects of the $1 \mathrm{~F} 2$ response may be suppressed or temporally integrated when disparity is zero in the adults or older children.

\section{Neural bases of disparity-orientation biases}

How might the qualitative difference in infant responses to different disparity orientations come about? One possibility is that the infant cortex has only a subset of the mechanisms that are present in the adult. On this view, two possible immaturities merit consideration. First, in the macaque central visual field, there is a strong bias in the range of disparity tuning for horizontal versus vertical disparity: the disparity range is larger for horizontal than vertical disparities (Cumming, 2002; Durand et al., 2007). In the periphery, however, the disparity ranges are similar for horizontal and vertical disparities, and both disparity ranges are larger than in the fovea (Durand et al., 2007). The extraction of $2 \mathrm{D}$ disparity has been modeled as a network property involving pooling across multiple disparity tuned subunits, combined with a normalization process (Read, 2010). Either or both of these processes could be immature in infants, given that the fovea is 
anatomically immature at 4-6 months in human infants (Hendrickson and Drucker, 1992).

Alternatively, or in addition to the central field bias in V1, it is possible that the differences in processing between horizontal and vertical disparities in early visual areas are further magnified in downstream extrastriate areas. A possible analogy for this viewpoint is the way in which responses to anticorrelated stimuli that are robust in V1 (Cumming and Parker, 1997) are eliminated in higher-order extrastriate areas (Parker, 2007). This model is attractive in that neither anticorrelated nor vertical disparity stereograms support a percept of depth, but both are capable of driving vergence eye movements (Masson et al., 1997; Stevenson et al., 1997). Vergence eye movements are already active well before the ages we have studied (Seemiller et al., 2016). It is thus possible that this substrate, which is not well understood (but see Takemura et al., 2007) is present in infants, but that other substrates, such as those that support depth-structure percepts are not.

\section{References}

Andrews TJ, Glennerster A, Parker AJ (2001) Stereoacuity thresholds in the presence of a reference surface. Vision Res 41:3051-3061. CrossRef Medline

Anzai A, Chowdhury SA, DeAngelis GC (2011) Coding of stereoscopic depth information in visual areas V3 and V3A. J Neurosci 31:1027010282. CrossRef Medline

Birch E, Petrig B (1996) FPL and VEP measures of fusion, stereopsis and stereoacuity in normal infants. Vision Res 36:1321-1327. CrossRef Medline

Birch EE, Salomão S (1998) Infant random dot stereoacuity cards. J Pediatr Ophthalmol Strabismus 35:86-90. Medline

Birch EE, Gwiazda J, Held R (1982) Stereoacuity development for crossed and uncrossed disparities in human infants. Vision Res 22:507-513. CrossRef Medline

Braddick O, Atkinson J (1983) The development of binocular function in infancy. Acta Ophthalmol Suppl 157:27-35. Medline

Braddick O, Atkinson J, Julesz B, Kropfl W, Bodis-Wollner I, Raab E (1980) Cortical binocularity in infants. Nature 288:363-365. CrossRef Medline

Brown AM, Lindsey DT, Satgunam P, Miracle JA (2007) Critical immaturities limiting infant binocular stereopsis. Invest Ophthalmol Vis Sci 48: 1424-1434. CrossRef Medline

Campbell FW, Maffei L (1970) Electrophysiological evidence for the existence of orientation and size detectors in the human visual system. J Physiol 207:635-652. CrossRef Medline

Chino YM, Smith EL 3rd, Hatta S, Cheng H (1997) Postnatal development of binocular disparity sensitivity in neurons of the primate visual cortex. J Neurosci 17:296-307. Medline

Cogan AI, Lomakin AJ, Rossi AF (1993) Depth in anticorrelated stereograms: effects of spatial density and interocular delay. Vision Res 33: 1959-1975. CrossRef Medline

Cogan AI, Kontsevich LL, Lomakin AJ, Halpern DL, Blake R (1995) Binocular disparity processing with opposite-contrast stimuli. Perception 24: 33-47. CrossRef Medline

Cottereau BR, McKee SP, Ales JM, Norcia AM (2011) Disparity-tuned population responses from human visual cortex. J Neurosci 31:954-965. CrossRef Medline

Cottereau BR, McKee SP, Norcia AM (2012a) Bridging the gap: global disparity processing in the human visual cortex. J Neurophysiol 107:24212429. CrossRef Medline

Cottereau BR, McKee SP, Ales JM, Norcia AM (2012b) Disparity-specific spatial interactions: evidence from EEG source imaging. J Neurosci 32: 826-840. CrossRef Medline

Cumming BG (2002) An unexpected specialization for horizontal disparity in primate primary visual cortex. Nature 418:633-636. CrossRef Medline

Cumming BG, Parker AJ (1997) Responses of primary visual cortical neurons to binocular disparity without depth perception. Nature 389:280283. CrossRef Medline

Cumming BG, Parker AJ (1999) Binocular neurons in V1 of awake monkeys are selective for absolute, not relative, disparity. J Neurosci 19:5602-5618. Medline

Dmochowski JP, Greaves AS, Norcia AM (2015) Maximally reliable spatial filtering of steady state visual evoked potentials. Neuroimage 109:63-72. CrossRef Medline

Durand JB, Celebrini S, Trotter Y (2007) Neural bases of stereopsis across visual field of the alert macaque monkey. Cereb Cortex 17:1260-1273. CrossRef Medline

Eizenman M, Westall CA, Geer I, Smith K, Chatterjee S, Panton CM, Kraft SP, Skarf B (1999) Electrophysiological evidence of cortical fusion in children with early-onset esotropia. Invest Ophthalmol Vis Sci 40:354-362. Medline

Erkelens CJ, Collewijn H (1985) Eye movements and stereopsis during dichoptic viewing of moving random-dot stereograms. Vision Res 25: 1689-1700. CrossRef Medline

Filippini HR, Banks MS (2009) Limits of stereopsis explained by local crosscorrelation. J Vis 9:8 1-18. CrossRef Medline

Harris JM, McKee SP, Smallman HS (1997) Fine-scale processing in human binocular stereopsis. J Opt Soc Am A Opt Image Sci Vis 14:1673-1683. CrossRef Medline

Held R, Birch E, Gwiazda J (1980) Stereoacuity of human infants. Proc Natl Acad Sci U S A 77:5572-5574. CrossRef Medline

Hendrickson A, Drucker D (1992) The development of parafoveal and mid-peripheral human retina. Behav Brain Res 49:21-31. CrossRef Medline

Jandó G, Mikó-Baráth E, Markó K, Hollódy K, Török B, Kovacs I (2012) Early-onset binocularity in preterm infants reveals experience-dependent visual development in humans. Proc Natl Acad Sci U S A 109:11049_ 11052. CrossRef Medline

Janssen P, Vogels R, Liu Y, Orban GA (2001) Macaque inferior temporal neurons are selective for three-dimensional boundaries and surfaces. J Neurosci 21:9419-9429. Medline

Janssen P, Vogels R, Liu Y, Orban GA (2003) At least at the level of inferior temporal cortex, the stereo correspondence problem is solved. Neuron 37:693-701. CrossRef Medline

Krug K, Parker AJ (2011) Neurons in dorsal visual area V5/MT signal relative disparity. J Neurosci 31:17892-17904. CrossRef Medline

Legge GE, Gu YC (1989) Stereopsis and contrast. Vision Res 29:989-1004. CrossRef Medline

Maruko I, Zhang B, Tao X, Tong J, Smith EL 3rd, Chino YM (2008) Postnatal development of disparity sensitivity in visual area 2 (v2) of macaque monkeys. J Neurophysiol 100:2486-2495. CrossRef Medline

Masson GS, Busettini C, Miles FA (1997) Vergence eye movements in response to binocular disparity without depth perception. Nature 389:283286. CrossRef Medline

McKee SP, Welch L, Taylor DG, Bowne SF (1990) Finding the common bond: stereoacuity and the other hyperacuities. Vision Res 30:879-891. CrossRef Medline

Mikó-Baráth E, Markó K, Budai A, Török B, Kovacs I, Jandó G (2014) Maturation of cyclopean visual evoked potential phase in preterm and fullterm infants. Invest Ophthalmol Vis Sci 55:2574-2583. CrossRef Medline

Norcia AM, Gerhard HE (2015) Development of three-dimensional perception in human infants. Annu Rev Vis Sc 1:569-594. CrossRef

Norcia AM, Hou C (2005) Random-dot stereopsis is highly immature in human infants. J Vis 5.

Norcia AM, Tyler CW, Hamer RD, Wesemann W (1989) Measurement of spatial contrast sensitivity with the swept contrast VEP. Vision Res 29: 627-637. CrossRef Medline

Norcia AM, McKee SP, Bonneh Y, Pettet MW (2005) Suppression of monocular visual direction under fused binocular stimulation: evoked potential measurements. J Vis 5:34-44. CrossRef Medline

Parker AJ (2007) Binocular depth perception and the cerebral cortex. Nat Rev Neurosci 8:379-391. CrossRef Medline

Pei F, Baldassi S, Tsai JJ, Gerhard HE, Norcia AM (2017) Development of contrast normalization mechanisms during childhood and adolescence. Vision Res 133:12-20. CrossRef Medline

Petrig B, Julesz B, Kropfl W, Baumgartner G, Anliker M (1981) Development of stereopsis and cortical binocularity in human infants: electrophysiological evidence. Science 213:1402-1405. CrossRef Medline

Read JC (2010) Vertical binocular disparity is encoded implicitly within a model neuronal population tuned to horizontal disparity and orientation. PLoS Comput Biol 6:e1000754. CrossRef Medline 
Seemiller ES, Wang J, Candy TR (2016) Sensitivity of vergence responses of 5- to 10-week-old human infants. J Vis 16:20. CrossRef Medline

Shiozaki HM, Tanabe S, Doi T, Fujita I (2012) Neural activity in cortical area V4 underlies fine disparity discrimination. J Neurosci 32:3830-3841. CrossRef Medline

Simons K (1981) Stereoacuity norms in young children. Arch Ophthalmol 99:439-445. CrossRef Medline

Stevenson SB, Lott LA, Yang J (1997) The influence of subject instruction on horizontal and vertical vergence tracking. Vision Res 37:2891-2898. CrossRef Medline

Takemura A, Murata Y, Kawano K, Miles FA (2007) Deficits in shortlatency tracking eye movements after chemical lesions in monkey cortical areas MT and MST. J Neurosci 27:529-541. CrossRef Medline
Tanabe S, Umeda K, Fujita I (2004) Rejection of false matches for binocular correspondence in macaque visual cortical area V4. J Neurosci 24:8170 8180. CrossRef Medline

Tang Y, Norcia AM (1995) An adaptive filter for steady-state evoked responses. Electroencephalogr Clin Neurophysiol 96:268-277. CrossRef Medline

Thomas OM, Cumming BG, Parker AJ (2002) A specialization for relative disparity in V2. Nat Neurosci 5:472-478. CrossRef Medline

Umeda K, Tanabe S, Fujita I (2007) Representation of stereoscopic depth based on relative disparity in macaque area V4. J Neurophysiol 98:241252. CrossRef Medline

Westheimer G (1979) Cooperative neural processes involved in stereoscopic acuity. Exp Brain Res 36:585-597. Medline 